\title{
Reflets
}

Revue ontaroise d'intervention sociale et communautaire

\section{Profil des ressources des communautés francophones en milieu minoritaire}

\section{Francine Deroche, Anne Simard, Erica Di Ruggiero et Johanne Levesque}

Volume 5, numéro 2, automne 1999

La santé des francophones de l’Ontario

URI : https://id.erudit.org/iderudit/026278ar

DOI : https://doi.org/10.7202/026278ar

Aller au sommaire du numéro

Éditeur(s)

Reflets : Revue ontaroise d'intervention sociale et communautaire

ISSN

1203-4576 (imprimé)

1712-8498 (numérique)

Découvrir la revue

Citer cet article

Deroche, F., Simard, A., Di Ruggiero, E. \& Levesque, J. (1999). Profil des

ressources des communautés francophones en milieu minoritaire. Reflets, 5(2),

202-212. https://doi.org/10.7202/026278ar

Tous droits réservés (C) Reflets : Revue ontaroise d'intervention sociale et communautaire, 1999
Ce document est protégé par la loi sur le droit d'auteur. L'utilisation des services d'Érudit (y compris la reproduction) est assujettie à sa politique d'utilisation que vous pouvez consulter en ligne.

https://apropos.erudit.org/fr/usagers/politique-dutilisation/ 


\title{
Profil des ressources des communautés francophones en milieu minoritaire ${ }^{1}$
}

\author{
F rancine D eroche'2, A nne Simard33, E rica D i R uggiero4, \\ Johanne Levesque ${ }^{5}$
}

\section{Introduction}

Le R éseau canadien de la santé (R C S) est une initiative nationale financée par Santé $C$ anada. Le $R$ éseau favorise l'accès à une information fiable, crédible et continuellement mise à jour, sur la santé et vient renforcer les réseaux existants de promotion de la santé. Fort du soutien et de l'apport d'organismes partenaires œuvrant aux quatre coins du pays, le $\mathrm{R} C \mathrm{~S}$ cherche à s'imposer auprès des $\mathrm{C}$ anadiens et $\mathrm{C}$ anadiennes comme source privilégiée de renseignements en matière de santé. Bien qu'il mise actuellement surtout sur Internet, le R CS espère offrir prochainement d'autres moyens d'accès à ces renseignements.

Le R CS a mené une enquête pour identifier quelles sont les informations disponibles sur la santé dans les communautés francophones vivant en milieu minoritaire ${ }^{6}$. II s'agit là d'un point de départ pour permettre au R CS de planifier et de mettre en œuvre des services en français pertinents. 


\section{Objectifs}

Les objectifs de l'enquête étaient:

- d'identifier et d'établir le profil des organismesqui traitent de la santé des Francophones;

- d'identifier les types d'organismes recommandés comme sources d'information aux consommateurs et consommatrices et aux intermédiaires de la santé;

- d'identifier les ressources existantes les plus souvent demandées par les consommateurset consommatrices et lesintermédiaires de la santé et,

- d'identifier les lacunes en matière d'informations sur la santé des francophones.

\section{Méthodologie}

C'est à partir de ces quatre objectifs d'enquête qu'on a conçu un questionnaire. La première partie du questionnaire, complétée au moyen d'entrevues téléphoniques, traitait de questions rattachées à l'information sur la santé: la clientèle, les types d'information, les questions importantes pour les francophones, les ressources les plus populaires, les lacunes d'information sur la santé des francophones, les moyens pour diffuser l'information auprès des francophones, les organismes clés dans le réseau d'information sur la santé et les stratégies visant à offrir des services d'information sur la santé. La deuxième partie du questionnaire, auto- administrée par les répondants et répondantes, dressait le profil desorganismes interviewés: le type d'organisme, les activités, les services, la clientèle et les modes d'accès.

Un comité a été créé. C elui-ci comprenait cinq membres, dont quatre représentaient une région du pays tandis que le cinquième incarnait une vision pancanadienne de la francophonie'. Le rôle du comité consistait à valider l'échantillon et le rapport final. L'enquête a été menée entre janvier et mars 1999. Le taux de réponse était $69 \%$ (soit 74 des 107 organismes identifiés). 


\section{Limites de l'enquête}

Q uoique l'échantillon comprenne une grande diversité d'organismes, il ne représente pas tous ceux qui desservent les francophones en matière d'informations sur la santé. Par exemple, plusieursorganismes bilingues ou ayant un rayonnement national ont été exclus de l'échantillon. C ertaines provinces et territoires sont peu ou pas représentés au sein de l'échantillon. De plus, l'échantillon n'a pas tenu compte du fait que les francophones vivant en milieu minoritaire doivent souvent fonctionner en anglais. II se peut donc qu'ils se tournent vers des organismes anglophones pour des demandes d'information.

U n cadre conceptuel a été créé afin de faciliter la tâche de l'analyse et de l'interprétation des données. Ce cadre théorique englobe les thèmes et les informations fournis par les répondants et répondantes. Les thèmes relèvent de quatre grandescatégories:

- contenus préas: desinformationsqui traitent de problématiques, de maladies ou d'états de santé précis ( $p$. ex. maladies chroniques, violence, santé mentale);

- stratégies et moyens: des informations qui traitent des moyens par lesquels on peut intervenir auprès d'une personne, d'un groupe ou d'une communauté ( $p$. ex. accès aux services, formation, financement);

- groupes cibles: des informations reliées à un groupe précis ou une population cible (p. ex. aînés, femmes, etc.);

- milieux: desinformationspar rapport à des lieux d'intervention précis (p. ex. santé publique et communautaire, milieu de travail, milieu scolaire).

Toutefois, si I'utilisation d'un cadre conceptuel aide à organiser les données, elle limite aussi les façons d'interpréter les données. 


\section{Constats}

Tout en tenant compte des limites de l'enquête, on peut établir plusieurs constats.

\section{Un échantillon diversifié}

D ans la mesure où l'enquête cherchait à rejoindre divers secteurs de la francophonie en milieu minoritaire, on peut dire: «mission accomplie ». Les répondants et les répondantes représentent plusieurs organismes de divers secteurs et de groupes : associations culturelles, santé communautaire et publique, femmes, éducation, services sociaux et psychosociaux, soins aigus, associations d'intervenants et d'intervenantes, gouvernements et autres. La majorité des organismes interviewés œuvrent à l'échelle provinciale, régionale ou locale.

\section{Différentes clientèles, avec davantage de services pour les consommateurs et les consommatrices}

Les organismes interviewés desservent autant les hommes que les femmes, bien que l'on note un plus grand nombre de femmes parmi leur clientèle. Ce fait ne devrait pas surprendre compte tenu du rôle des femmes en matière de santé.

Les organismes offrent davantage des services directs à la population qu'à des intermédiaires de la santé, comme les groupes communautaires, les professionnels de la santé, les organismes de la santé, les organismes d'action bénévole et les intervenants et intervenantes en promotion de la santé. Ce faisant, on peut se demander si ces organismes connaissent bien ou sils peuvent répondre aux besoins d'information des intermédiaires. Sans préjuger des faits, il faudrait pouvoir regarder cette question de plus près. 


\section{Un réseau provincial, régional et local en santé et services communautaires}

U ne analyse des principaux organismes, vers lesquels on oriente les gens désirant obtenir de l'information sur la santé des francophones, confirme qu'il y a peu d'acteurs et d'actrices de la scène nationale. Ainsi, le réseau d'information revêt largement un caractère provincial, régional, voire local. C ela étant dit, les limites de l'enquête peuvent aussi expliquer ce résultat.

L'enquête n'a pas permis d'identifier avec certitude les têtes de réseaux, mais elle procure des renseignements utiles propres à identifier les organismes qui ont un rôle important en matière de services d'information sur la santé en français. Ces organismes sont largement associés au secteur de la santé et des services communautaires, comme le sont les centres de santé communautaire et les bureaux de santé publique, pour prendre un exemple ontarien.

Si certains organismes québécois ont été mentionnés comme source principale d'information en français sur la santé, cela vient souligner le rôle du Q uébec comme pôle de référence pour le milieu minoritaire. $M$ ais il ne faudrait pas exagérer ce constat. $E n$ effet, il faut souligner le peu de références faites aux organismes québécois, puisqu'ils ont été identifiés seulement huit fois parmi nos 149 réponses. O n peut possiblement attribuer un tel résultat aux besoins particuliers qu'éprouvent les gensdemeurant en milieu minoritaire et aux difficultés de transfert d'un système provincial à un autre.

\section{Des ressources existantes davantage axées sur l'accès aux services et ressources}

La tendance est semblable pour les sources d'informations Internet. Les sources les plus populaires concernent l'accès et le développement de ressources ou l'accès aux services. $\mathrm{Ce}$ phénomène s'explique par le fait que les francophones en milieu minoritaire sont souvent à la recherche de services et de ressources en français. 


\section{Un manque de ressources en matière de contenu précis et de stratégies}

En général, les consommateurs et les consommatrices francophones demandent le plus souvent de l'information sur la santé ayant des contenus précis. C ette tendance est aussi vrai chez les groupes intermédiaires, bien que leur demande porte sur un nombre restreint de sujets. Par exemple, la santé mentale et la toxicomanie occupent l'avant- scène des demandes d'informations des consommateurs et des consommatrices francophones. $0 \mathrm{n}$ observe aussi la même tendance chez les intermédiaires.

Les stratégies et les moyens sont la deuxième information la plus so uvent demandée chez les intermédiaires, tandis qu'elle est la troisième chez les consommateurs et les consommatrices. $C$ hez les intermédiaires, une des demandes majeures a trait à l'accès aux ressources et à la stratégie d'action communautaire, car ils y voient un moyen leur permettant d'intervenir efficacement auprès de leurs clientèles.

Les lacunes de ressources identifiées viennent confirmer en grande partie les résultats d'étude sur les demandes d'information. En premier lieu, les principales lacunes sont d'ordre pratique et résident au niveau des stratégies et des moyens, suivis en cela par de l'information sur des contenus précis. A u niveau des stratégies et des moyens, le domaine prioritaire à combler est celui de l'accès aux ressources.A u niveau des contenus, les priorités doivent porter sur la santé mentale et la sexualité. L'ajout de la dimension «santé mentale » apporte ici une nuance intéressante par rapport aux demandes d'information qui, elles, mettaient principalement l'accent sur la sexualité.

Les ressources existantes les plus populaires ne concordent pas nécessairement aux demandes d'informations des consommateurs ou des consommatrices et des intermédiaires de la santé. C omme on l'a vu auparavant, les demandes traitent en grande majorité de contenus précistandisque les ressourcestraitent largement des stratégies et moyens. 


\section{Des nouvelles questions de santé qui reflètent les tendances sociales et les préoccupations d'une communauté minoritaire}

Par ordre de priorité, les questions de santé qui préoccupent premièrement les francophones portent sur des contenus précis, comme la santé mentale. $\mathrm{O}$ n devra donc trouver un moyen de leur fournir de l'information dans les prochaines années. L'information sur la sexualité est également identifiée comme comportant des lacunes importantes.

En second lieu, les groupes cibles occupent aussi une grande place dans les demandes d'information, notamment les personnes âgées et les femmes. C ette tendance est le reflet de notre société dans la mesure où la population est vieillissante et les femmes sont appelées à jouer un rôle important à titre de soignantes.

Troisièmement, les demandes d'informations portent sur les stratégies et les moyens. I ci encore, il s'agit d'une tendance sociale que favorise un virage vers la promotion de la santé où l'accent est mis sur le choix de stratégies prioritaires. D e plus, on souligne de nouveau l'accès aux services, sujet qui est revenu à maintes reprises dans le cadre de cette enquête.

\section{Un réseau naturel pour l'élaboration et la distribution d'informations}

La diffusion d'information et de ressources est un service offert par la grande majorité des répondants. De plus, nombreux sont ceux qui distribuent des informations produites par d'autres organismes. C'est donc dire qu'on peut accéder à bon nombre d'organismes existants pour diffuser de l'information et sensibiliser la francophonie en milieu minoritaire aux services existants. De plus, $67 \%$ des organismes interviewés élaborent du matériel. II s'agit donc d'une autre possibilité pour appuyer le développement de contenus pertinents pour la francophonie en milieu minoritaire.

La majorité des répondants et répondantes possède une ligne téléphonique d'information et un site web. C e fait est intéressant parce qu'il offre la possibilité de diffuser l'information par le biais 
des réseaux existants. Cependant, plus de $40 \%$ des répondants n'ont pas de site web. De plus, selon une enquête récente de CyberTRENDS, le taux d'internautes hebdomadaires francophones est inférieur au taux anglophone ( $23 \%$ vs. $38 \%)^{8}$. II faut donc prévoir une variété de modes d'accès à l'information ou encore, appuyer les organismes dans le développement de leurs capacités technologiques. $\mathrm{N}$ otons par ailleurs que les organismes en éducation, culture et santé communautaire sont ceux qui possèdent un site web.

\section{Une culture qui aime parler : un autre indice pour diffuser l'information}

Le face-à-face est le moyen le plus efficace pour diffuser l'information sur la santé sel on les répondants et les répondantes. Les gens s'intéressent à Internet et le trouvent pertinent comme outil de diffusion. Cependant, on doit aussi tenir compte des autres moyens de diffusion, comme les médias de masse surtout, les médias communautaires et les médias à public restreint. $\mathrm{Ce}$ constat renforce l'importance d'assurer l'accès à l'information par divers modes, de bâtir sur les acquis en accédant aux réseaux locaux et régionaux, et d'adapter l'information sur le plan régional ou local.

Selon I'outil Si je savais comment... R ejoindre les $F$ rancophones, faut l'faire! !', il faut identifier quels véhicules de communication et de réseaux de distribution de l'information conviendraient le mieux à sa communauté d'intérêt. Par exemple, dans le cas de I'O ntario français, nous savons que la transmission de bouche à oreille est très efficace alors que la communication de masse n'est pas vraiment une solution étant donnée le peu d'infrastructure existante. II faut donc miser davantage sur la vaste infrastructure de communication qui existe à l'échelle communautaire. II s'agit ici d'un réseau communautaire organisé, comme la Fédération des caisses populaires de l'O ntario ou encore d'un réseau socio-communautaire informel, comme les clubs d'âge d'or. 


\section{9. À la recherche de fondements solides pour offrir des services pertinents à une communauté minoritaire}

Bon nombre de stratégies et de moyens ont été proposés pour aider le R C S à donner des services pertinents et des ressources supplémentaires pour les francophones. Les communications y figurent au premier plan afin de sensibiliser, diffuser, traduire et adapter les ressources existantes. L'intérêt qu'on démontre face à l'accès aux ressources confirme de nouveau l'appétit pour divers modes d'accès. O n soulignel'importance du réseautage en utilisant les réseaux existants, et en misant tout particulièrement sur les partenariats.

Et, puisqu'on dressait le profil d'une communauté minoritaire, les questions qui préoccupent la clientèle, composée de consommateurs, de consommatricesou d'intermédiaires, touchent souvent l'accès aux services médicaux et préventifs de base en français. Par ailleurs, il est clair que la sensibilisation et la revendication de services en français préoccupent toujours les organismes francophones.

De la même façon, certaines régions du pays sont mieux desservies que d'autres. Si un réseau de centres de santé communautaire francophones existe en 0 ntario, en Alberta, on est surtout préoccupé par le besoin de trouver des intervenants francophones en A lberta. C eci dépend de plusieurs facteurs, tels le nombre de francophones dans la région ou la capacité de la communauté francophone de revendiquer ses droits. De plus, le profil socio-économique des groupes et des régions francophones en milieu minoritaire est loin d'être homogène. D eux exemples illustreront ce manque d'homogénéité. Premièrement, il y a les francophones de souche qui se trouvent dans les régions traditionnelles. Si ces derniersmaintiennent assez bien leurs acquis sur les plans linguistique et culturel, ils sont souvent confrontés à des problèmes économiques. Deuxièmement, il y a les francophones vivant en situation minoritaire dans les régions urbaines anglophones. C eux-ci sont plus aisés sur le plan économique, mais ils sont davantage sujets au processus 
d'assimilation et ce, à un rythme plus rapide (Institut canadien de recherche sur le développement régional, 1994). Ce manque d'homogénéité de la communauté francophone dicte le besoin d'avoir une approche régionale pour combler les besoins.

Les francophones du C anada font face à d'autres difficultés. De nombreuses communautés francophones dans les régions éloignées souffrent d'isolement. Les francophones sont dispersés et n'arrivent pasà obtenir une cohésion sociale et communautaire francophone. $\mathrm{O}$ affiche des taux élevés d'analphabétisme. L'assimilation menace toujours la survie de la langue française et de la culture francophone. Et de nombreux francophones n'ont pas accès aux services de santé en français, malgré le fait que la langue est à la base d'une prestation de services de santé adéquats et contribue largement au succès des relations entre le client ou la cliente et le fournisseur ou la fournisseuse de services de santé.

\section{Conclusion}

Ce profil de ressources permettra l'élaboration d'une stratégie qui saura offrir et faciliter l'accès à des services et desinformations qui répondent aux besoins de la francophonie canadienne. En tant que «réseau de réseaux », le R C S pourra continuer son rôle rassembleur ou de fil conducteur et collaborer avec les réseaux existants pour offrir des services d'informations sur la santé en français. De par son mandat, le R C S continuera la collecte de données pour la francophonie en milieu minoritaire, tant au niveau des divers types de ressources disponibles qu'au niveau des têtes de réseaux.

\section{Bibliographie}

IN ST ITUT CANADIEN DE RECHER CHE SUR LE DÉVELOPPEMENT RÉGIONAL (1994). État de la francophonie hors Q uébec en 1991, 0 ttawa, Fédération des communautés francophones et acadienne du C anada. 


\section{Notes}

1. On peut obtenir une copie du rapport de cette enquête en communiquant avec les auteures.

2. Consultante, IN N OVACTIO N , 180, rue Dundas O uest, bureau 1900,Toronto (O ntario) M 5 G $1 Z 8$ http:/ / www.innovaction.com, téléphone: (416) 408-4499 ou 1-888-innovac, télécopieur: (416) 408-4716, courrier électronique : francine@ innovaction.com ou anne@ innovaction.com

3. Consultante, IN N OVACT IO N

4. Gestionnaire du développement des partenariats opérationnels, réseau canadien de la santé, 180, rue D undas 0 uest, bureau 1700,Toronto (O ntario) M 5 G 1Z8, http:/ / ww w.reseau-canadiensante.ca, téléphone: (416) 408-4054 ou 1-877-891-IN FO, télécopieur: (416) 408-4394, courrier électronique : ericadi@innovaction.com ou Johanne_Levesque@ hc-sc.gc.ca

5. Gestionnaire des partenariats stratégiques, réseau canāien de la santé

6. Par les communautés francophones en milieu minoritaire, on entend les près d'un million de francophones demeurant à l'extérieur du Q uébec.

7. Les membres du comité étaient: Gilles R enault, R éseauN at; Susan Lilley, Le R éseau pour la promotion de la santé communautaire; Louise Picard, Service de santé publique de Sudbury et du district; Anne Leis, U niversité de Saskatchewan; Penny M osmann, Bureau régional de Santé Canada.

8. Édition printemps 1999 du sondage CyberTrends produit par ComQ uest R esearch. $M$ alheureusement il n'y a pas de données disponibles pour établir des comparai sons entre les francophones des différentes régions du pays. D onnées fournies par Industrie C anada.

0 rdinateurs à domicile (pou rcentage des adultes canadiens [18+ ] par langue)

Anglophones: $\quad 57 \%$ (10,03 millions)

Francophones: $\quad 42 \%$ (2,52 millions)

Autres: $\quad 59 \%$ (0,50 millions)

Internautes hebdomadaires (pourcentage des adultes canadiens [18+] par langue)

D éc-97 M ars-98 D éc-1998 M ars-99

A nglophones : $26 \% \quad 28 \% \quad 32 \% \quad 38 \%$

\begin{tabular}{|c|c|c|c|c|}
\hline \multicolumn{2}{|c|}{ Francophones : $14 \%$} & \multirow[b]{2}{*}{$18 \%$} & \multirow[b]{2}{*}{$27 \%$} & (6,71 millions) \\
\hline F rancopl & : $14 \%$ & & & $\begin{array}{l}23 \% \\
\text { (1,39 millions) }\end{array}$ \\
\hline A utres: & $28 \%$ & $38 \%$ & $39 \%$ & $\begin{array}{l}32 \% \\
(0,27 \text { millions })\end{array}$ \\
\hline
\end{tabular}

9. C e document a été réalisé en 1995 par la firme $N$ adeau, Beaulieu etA ssocié.e.s en collaboration avec le $\mathrm{C}$ omité encadreur du Service de la santé d'O ttawa-C arleton. 